\title{
MicroRNA-200b inhibits the growth and metastasis of glioma cells via targeting ZEB2
}

\author{
JIAN LI ${ }^{1,3}$, JIAN YUAN $^{1}$, XIANRUI YUAN ${ }^{1}$, JIE ZHAO $^{1,3}$, ZHIPING ZHANG $^{1,3}$, \\ LING WENG $^{2}$ and JINGPING LIU ${ }^{1,3}$ \\ Departments of ${ }^{1}$ Neurosurgery, ${ }^{2}$ Neurology, ${ }^{3}$ Hydrocephalus Center, Xiangya Hospital, \\ Central South University, Changsha, Hunan 410008, P.R. China
}

Received September 6, 2015; Accepted October 27, 2015

DOI: $10.3892 /$ ijo.2015.3267

\begin{abstract}
MicroRNAs (miRs) have been found to play important roles in mediating a variety of biological processes in human cancers, including tumor cell proliferation, migration, invasion, and epithelial-mesenchymal transition (EMT). In the present study, we aimed to investigate the putative role of miR-200b in the progression of glioma. Real-time RT-PCR data showed that the miR-200b levels were frequently reduced in primary glioma tissues $(n=88)$ and cell lines, when compared to normal brain tissues $(n=25)$. Moreover, decreased miR-200b level was tightly associated with the malignant progression of glioma. Overexpression of miR-200b significantly suppressed cell proliferation, migration, invasion and EMT in glioma U251 and U87 cells. Luciferase reporter assay data further identified ZEB2 as a direct target of miR-200b, and the protein expression of ZEB2 was markedly reduced after overexpression of miR-200b in U251 and U87 cells. Furthermore, restoration of ZEB2 effectively reversed the reduced expression of ZEB2, as well as the suppressive effects of miR-200b overexpression on the proliferation, migration, invasion and EMT in glioma U251 and U87 cells. Moreover, in vivo study showed that overexpression of miR-200b significantly inhibited tumorigenesis as well as the tumor growth of glioma cells, and effectively protected nude mice from tumor-induced death. Taken together these findings suggest that miR-200b has suppressive effects on the proliferation, migration, invasion and EMT of glioma cells, partly at least, via targeting ZEB2. Therefore, miR-200b acts as a novel tumor suppressor in glioma, and thus may become a promising therapeutic candidate for glioma.
\end{abstract}

Correspondence to: Professor Jingping Liu, Department of Neurosurgery, Xiangya Hospital, Central South University, Xiangya Road 87, Changsha, Hunan 410008, P.R. China

E-mail: csuliujingping@sina.com

Key words: glioma, microRNA, ZEB2, proliferation, metastasis, epithelial-mesenchymal transition

\section{Introduction}

Glioma, the most common of brain tumors, accounts for $\sim 30 \%$ of central nervous system tumors and $80 \%$ of all malignant brain tumors (1). Despite the remarkable development of therapies for other cancers, the median survival rate of glioma has not been remarkably improved over the past few decades, mainly due to its resistance to radiotherapy, chemotherapy and adjuvant therapies (2-5). Therefore, a more effective therapeutic strategy is urgently needed.

MicroRNAs (miRs), a kind of short non-coding RNA, generally lead to mRNA degradation or inhibition of protein translation, through directly binding to the 3 ' untranslational region (3'UTR) of their target mRNAs (6). It has been well established that miRs play important roles in the regulation of various biological processes, including cell proliferation, differentiation, apoptosis, cell cycle progression, and migration (7). Furthermore, both the upregulation of oncogenic miRs and the downregulation of tumor suppressor miRs are involved in tumorigenesis and cancer metastasis $(8,9)$. Recent studies have suggested that miR-200b is frequently downregulated in glioma, and the decreased miR-200b expression is an independent unfavorable prognostic factor for glioma patients $(10,11)$. Besides, miR-200b was reported to play a suppressive role in the regulation of glioma cell growth via targeting cAMP responsive element-binding protein 1 (CREB1) (12). However, the exact role of miR-200b in the progression of glioma as well as the underlying mechanism still remain to be fully uncovered.

ZEB2, a member of the Zfh1 family of 2-handed zinc finger/homeodomain proteins, is located in the nucleus and functions as a DNA-binding transcriptional repressor that interacts with activated Shades (13). Epithelial-mesenchymal transition (EMT) plays a key role in cancer cell metastasis $(14,15)$. During EMT, E-cadherin is upregulated, while vimentin and $\mathrm{N}$-cadherin are downregulated (16). Recent studies have found that ZEB2 can bind to the E-cadherin promoter, and suppress the expression of E-cadherin during EMT (13). Moreover, ZEB2 is also reported to promote the transcription of vimentin via cooperation with Sp1 during EMT (17). Therefore, ZEB2 plays a promoting role in EMT. Moreover, our previous study demonstrated that ZEB2 is significantly increased in glioma tissues, and acts as an 
oncogene via mediating cell proliferation, cell cycle progression, apoptosis, migration, invasion and EMT in glioma (18). However, the regulatory mechanism of ZEB2 in glioma remains largely unclear.

In the present study, we investigated the putative role of miR-200b in the progression of glioma. We found that the expression of miR-200b was frequently reduced in clinical glioma tissue samples compared to normal brain tissues, and decreased miR-200b level is tightly correlated with the malignant progression, suggesting that miR-200b acts as a tumor suppressor in glioma. We further identified ZEB2 as a direct target gene of miR-200b. In vitro investigation revealed that miR-200b could inhibit the proliferation, migration, invasion and EMT of glioma cells through inhibition of ZEB2 expression.

\section{Materials and methods}

Ethics statement. The study was approved by the Ethics Committee of Central South University, Changsha, China, and written informed consents were obtained from all studied patients.

Clinical specimens. A total of 88 cases of glioma specimens and 25 normal brain tissues were obtained from Xiangya Hospital of Central South University between June 2011 and July 2013. The glioma patients included 40 female and 48 male who ranged in age from 20 to 77 years, with a mean of 47.2 years. The patients underwent MRI a few days before and within $72 \mathrm{~h}$ after surgery, and the extent of tumor resection was determined using the postoperative MRI scans. None of the patients received either radiation therapy or chemotherapy before surgical resection. All specimens had confirmed pathological diagnosis and were classified according to the World Health Organization (WHO) criteria. Among the 88 glioma samples, 34 were classified as low-grade including 10 pilocytic astrocytomas (WHO I) and 24 diffuse astrocytomas (WHO II), and 54 were classified as high-grade gliomas including 21 anaplasia astrocytomas (WHO III), and 33 primary glioblastomas (WHO IV). Clinicopathological information of the patients is summarized in Table I. All tissue samples were immediately snap-frozen in liquid nitrogen and stored at $-80^{\circ} \mathrm{C}$ until use.

Cell lines. Normal human astrocytes (NHA) and human glioma cell lines U251 and U87 were purchased from the Chinese Academy of Sciences (Shanghai, China). Cells were cultured in Dulbecco's modified Eagle's medium (DMEM) supplemented with $10 \%$ fetal bovine serum (FBS) (both from Life Technologies), $100 \mathrm{IU} / \mathrm{ml}$ penicillin and $100 \mathrm{IU} / \mathrm{ml}$ streptomycin. Cells were cultured at $37^{\circ} \mathrm{C}$ in a humidified atmosphere with $5 \% \mathrm{CO}_{2}$.

Real-time RT-PCR assay. Total RNA was extracted by using TRIzol reagent (Life Technologies). For detection of miRs, a miRNA Reverse Transcription kit (Life Technologies) was used to convert RNA into cDNA, according to the manufacturer's instructions. Real-time PCR was then performed by using a miRNA qPCR detection kit (GeneCopoeia, Rockville, MD, USA) on ABI 7500 thermocycler. U6 gene was used as an internal reference. For mRNA detection, Reverse Transcription kit (Life Technologies) was used to convert RNA into cDNA, according to the manufacturer's instruction. Real-time PCR was then performed by using qPCR detection kit (Life Technologies) on ABI 7500 thermocycler. The primers used were the following: miR-200b forward, 5'-GCCGCTAATA CTGCCTGGTAATG-3' and reverse, 5'-GTGCAGGGTTCC GAGGT-3'; U6 forward, 5'-CGCTTCGGCAGCACATATA-3' and reverse, 5'-TTCACGAATTTGCGTGTCAT-3'. The PCR steps were $95^{\circ} \mathrm{C}$ for $10 \mathrm{~min}$, and 40 cycles of denaturation at $95^{\circ} \mathrm{C}$ for $15 \mathrm{sec}$ and annealing/elongation step at $60^{\circ} \mathrm{C}$ for $60 \mathrm{sec}$. The relative miR-200b expression was normalized to U6. The relative expression was analyzed by the $2^{-\Delta \Delta \mathrm{Ct}}$ method (19).

Western blot analysis. Cells were lysed with ice-cold lysis buffer (50 mM Tris-HCl, pH 6.8, $100 \mathrm{mM}$ 2-ME, 2\% w/v SDS, $10 \%$ glycerol). After centrifugation at 20,000 $\mathrm{xg}$ for $10 \mathrm{~min}$ at $4^{\circ} \mathrm{C}$, proteins in the supernatants were quantified and separated with $10 \%$ SDS-PAGE. Then, proteins were transferred onto a polyvinylidene difluoride (PVDF) membrane (Amersham Bioscience, Buckinghamshire, UK), which was then incubated with PBS containing $5 \%$ milk overnight at $4^{\circ} \mathrm{C}$. The PVDF membrane was incubated with rabbit anti-ZEB2 polyclonal antibody (1:200), rabbit anti-E-cadherin monoclonal antibody $(1: 200)$, rabbit anti-N-cadherin polyclonal antibody (1:200), rabbit anti-vimentin monoclonal antibody (1:100), and rabbit anti-GAPDH polyclonal antibodies (1:200) at room temperature for $3 \mathrm{~h}$, respectively, and then with HRP-linked goat anti-rabbit secondary antibody $(1: 10,000)$ (all from Abcam, Cambridge, MA, USA) at room temperature for $1 \mathrm{~h}$. SuperSignal West Pico Chemiluminescent Substrate kit (Pierce, Rockford, IL, USA) was then used to detect signals, according to the manufacturer's instructions. The relative protein expression was analyzed by Image-Pro plus software 6.0 , presented as the density ratio vs. GAPDH.

Plasmid constructions. The full-length 3'UTR of ZEB2 was amplified from human genomic DNA, and then cloned into the downstream of the firefly luciferase coding region of pMiR-GLO ${ }^{\mathrm{TM}}$ Luciferase vector (Promega, Madison, WI, USA), named as pMiR-ZEB2. Mutations of miR-200b binding sites were introduced by site-directed mutagenesis, which was then cloned downstream of the firefly luciferase coding region of pMiR-GLO ${ }^{\mathrm{TM}}$ Luciferase vector, named pMiR-Mut ZEB2.

Small interfering RNAs, miR mimic and inhibitor. The pcDNA3.1-ZEB2 plasmid, miR-200b mimic, and scramble miR mimic, were purchased from Nlbio (Changsha, China).

MTT assay. The 3-(4,5-dimethylthiazol-2-yl)-2,5-diphenyltetrazolium bromide (MTT) assay was used to examine cell proliferation (20). Briefly, cells in each group were plated at a density of 10,000 cells/well in 96-well plates. After cultured for 12, 24, 48 and $72 \mathrm{~h}$, the cells were incubated with MTT at a final concentration of $0.5 \mathrm{mg} / \mathrm{ml}$ for $4 \mathrm{~h}$ at $37^{\circ} \mathrm{C}$. After the removal of the medium, $150 \mathrm{mM}$ DMSO solutions were added to dissolve the formazan crystals. The absorbance was read at $570 \mathrm{~nm}$ using a multi-well scanning spectrophotometer reader. Cells in the control group were considered $100 \%$ viable. 
Cell migration assay. Wound healing assay was performed to evaluate the cell migratory capacity of glioma cells in each group. In brief, cells were cultured to full confluence. Wounds of $\sim 1 \mathrm{~mm}$ width were created with a plastic scriber, and cells were washed and incubated in a serum-free medium. After wounding for $24 \mathrm{~h}$, cells were incubated in a medium including $10 \%$ FBS. After cultured for $48 \mathrm{~h}$, cells were fixed and observed under a microscope.

Cell invasion assay. Cell invasion assay was performed using Transwell chambers (BD Biosciences, Franklin Lakes, NJ, USA), which were pre-coated with Matrigel. Cell suspension containing $5 \times 10^{5}$ cells $/ \mathrm{ml}$ was prepared in serum-free media, and $300 \mu \mathrm{l}$ of cell suspension was added into the upper chamber. Then, $500 \mu \mathrm{l}$ of DMEM with $10 \%$ FBS was added into the lower chamber. Cells were incubated for $24 \mathrm{~h}$. A cotton-tipped swab was used to carefully remove the cells that did not migrate or invade through the pores. The filters were fixed in $90 \%$ alcohol and stained by crystal violet, and observed under an inverted microscope (Olympus, Tokyo, Japan). After that, $0.5 \mathrm{~g} / \mathrm{l}$ MTT was added and incubated at $37^{\circ} \mathrm{C}$ for $4 \mathrm{~h}$. Then, the medium containing MTT was removed, and $50 \mu \mathrm{l}$ of dimethylsulfoxide was added to each well. Following incubation at $37^{\circ} \mathrm{C}$ for $10 \mathrm{~min}$, the optical density at $570 \mathrm{~nm}$ was measured using the Bio-Tek $^{\mathrm{TM}}$ ELX-800 ${ }^{\mathrm{TM}}$ Absorbance Microplate reader.

Luciferase reporter gene assay. Cells were seeded into 24-well plates and co-transfected with $200 \mathrm{ng}$ of pMiR-ZEB2 or pMiR-ZEB2-Mut vector and $100 \mathrm{ng}$ of miR-200b mimic or scramble miR mimic, and the pRL-TK plasmid (Promega) as internal normalization. Cells were harvested after $36 \mathrm{~h}$ and lysed using the lysis buffer (Promega). Luciferase reporter gene assay was conducted by using the Dual-Luciferase reporter assay system (Promega), in accordance with the manufacturer's instruction.

Stable transfection and tumor growth analysis. Male BALB/ $\mathrm{C}$-nu/nu nude mice ( 8 weeks) were purchased and maintained under specific pathogen-free condition at the Animal Center of Central South University. The miR-200b was cloned into the pLVX-IRES-ZsGreen1 vector (Nlbio) to construct the pYr-LVX-miR-200b lentiviral plasmid. U251 cells were then stably transfected with pYr-LVX-miR-200b lentiviral plasmid or blank pLVX-IRES-ZsGreen1 vector as controls. To determine the effect of miR-200b on the tumorigenesis of glioma cells in vivo, nude mice $(\mathrm{n}=6)$ were injected subcutaneously in the dorsal flank with $5 \times 10^{6}$ U251 cells stably transfected with miR-200b-overexpressing plasmid. Besides, in the control group, nude mice $(n=6)$ were injected with $5 \times 10^{6}$ U251 cells stably transfected with the blank vector. Survival time was recorded. The surviving nude mice were sacrificed 60 days after tumor implantation. Tumor volume was calculated by using the formula $\mathrm{V}\left(\mathrm{mm}^{3}\right)=0.5 \mathrm{xaxb}^{2}$ (a maximum length to diameter, $\mathrm{b}$ maximum transverse diameter), and the tumor weight was recorded.

Statistical analysis. Data were expressed as mean \pm standard deviation from at least three separate experiments. SPSS 18.0 software was used to perform statistical analysis. Comparisons of real-time data were analyzed by the unpaired t-test,
Table I. Correlation between 200b expression and clinicopathological features of glioma patients.

\begin{tabular}{|c|c|c|c|c|}
\hline \multirow[b]{2}{*}{$\begin{array}{l}\text { Clinicopathological } \\
\text { features }\end{array}$} & \multirow[b]{2}{*}{$\begin{array}{l}\text { Cases } \\
\text { (n) }\end{array}$} & \multicolumn{2}{|c|}{ 200b expression } & \multirow[b]{2}{*}{ P-value } \\
\hline & & $\begin{array}{l}\text { High } \\
\text { n }(\%)\end{array}$ & $\begin{array}{l}\text { Low } \\
\mathrm{n}(\%)\end{array}$ & \\
\hline \multicolumn{5}{|l|}{ Gender } \\
\hline Male & 48 & $21(43.8)$ & $27(56.3)$ & NS \\
\hline Female & 40 & $14(35)$ & $26(65)$ & \\
\hline \multicolumn{5}{|l|}{ Age (years) } \\
\hline$\leq 47$ & 42 & $18(42.9)$ & $24(57.1)$ & NS \\
\hline$>47$ & 46 & $17(37.0)$ & $29(63.0)$ & \\
\hline $\begin{array}{l}\text { Tumor size } \\
\text { (diameter) } \\
\leq 5 \mathrm{~cm} \\
>5 \mathrm{~cm}\end{array}$ & & $\begin{array}{l}(40.4) \\
(39.0)\end{array}$ & $\begin{array}{l}28(59.6) \\
25(61.0)\end{array}$ & NS \\
\hline $\begin{array}{l}\text { WHO g } \\
\text { I and I } \\
\text { III and }\end{array}$ & 54 & $\begin{array}{c}31(91.2) \\
4(7.4)\end{array}$ & $\begin{array}{c}3(8.8) \\
50(92.6)\end{array}$ & 0.0001 \\
\hline $\begin{array}{l}\leq 98 \% \\
>98 \%\end{array}$ & $\begin{array}{l}31 \\
57\end{array}$ & $\begin{array}{l}12(38.7) \\
23(40.4)\end{array}$ & $\begin{array}{l}19(61.3) \\
34(59.6)\end{array}$ & NS \\
\hline
\end{tabular}

whereas qualitative data were analyzed by the Chi-square test. Correlation was determined by Pearson correlation analysis. $\mathrm{P}<0.05$ were considered statistically significant.

\section{Results}

Analysis of miR-200b expression and its association with clinicopathological features of gliomas. To reveal the role of miR-200b in glioma, we firstly conducted real-time RT-PCR to examine the expression levels of miR-200b in 88 cases of glioma tissues as well as 25 cases of normal brain tissues as controls. The miR-200b levels were frequently decreased in glioma tissues compared to normal brain tissues (Fig. 1A). Besides, the expression level of miR-200b was also decreased in glioma U251 and U87 cells (Fig. 1B).

We further divided glioma patients into two groups, high miR-200b level and low miR-200b level, according to the median value of the miR-200b expression as the cutoff point. Among all glioma patients, 53 (60.23\%) were in low miR-200b level group, and 35 (39.77\%) were in high miR-200b level group. Based on these two groups, we analyzed the association of miR-200b levels with clinicopathological features of human gliomas. As shown in Table I, the high-grade glioma tissues (grade III and IV) showed lower miR-200b levels compared to the low-grade glioma tissues (grade I and II) $(\mathrm{P}<0.001)$. Moreover, no statistically significant association of miR-200b expression was found with the age, gender, tumor size, and extent of resection (both $\mathrm{P}>0.05$ ) (Table I). Based on these data, we suggest that aberrant downregulation of miR-200b may play a key role in the progression of human glioma. 

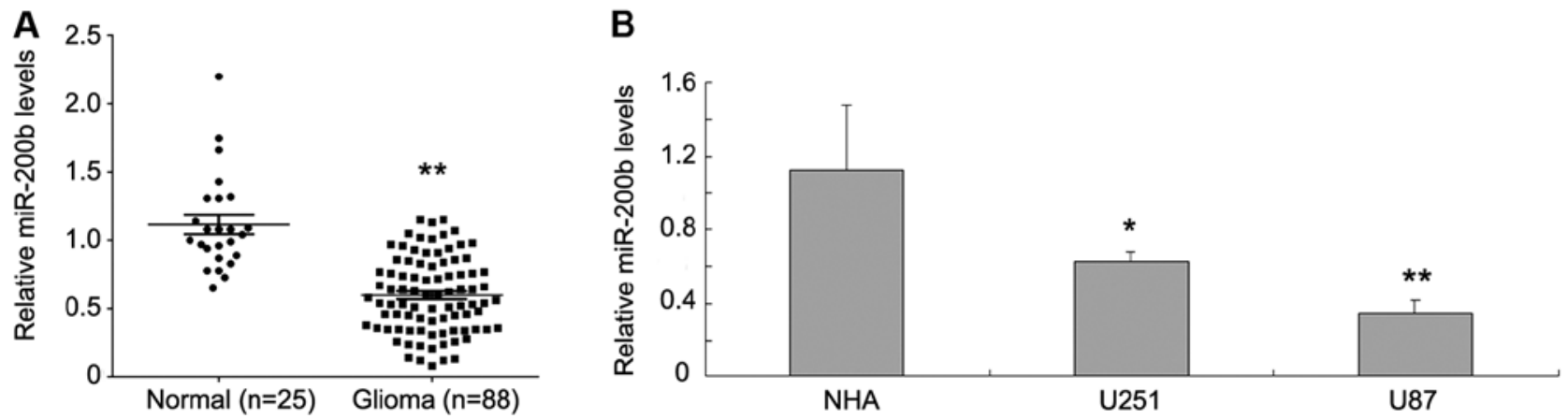

Figure 1. miR-200b expression in glioma tissues and cells. (A) Real-time RT-PCR was conducted to determine the relative miR-200b level in 25 cases of normal brain tissues and 88 cases of primary glioma tissues. (B) Real-time RT-PCR was conducted to determine the relative miR-200b level in normal human astrocytes (NHA) and glioma U251 and U87 cell lines. ${ }^{*} \mathrm{P}<0.05$ and ${ }^{* *} \mathrm{P}<0.01$.

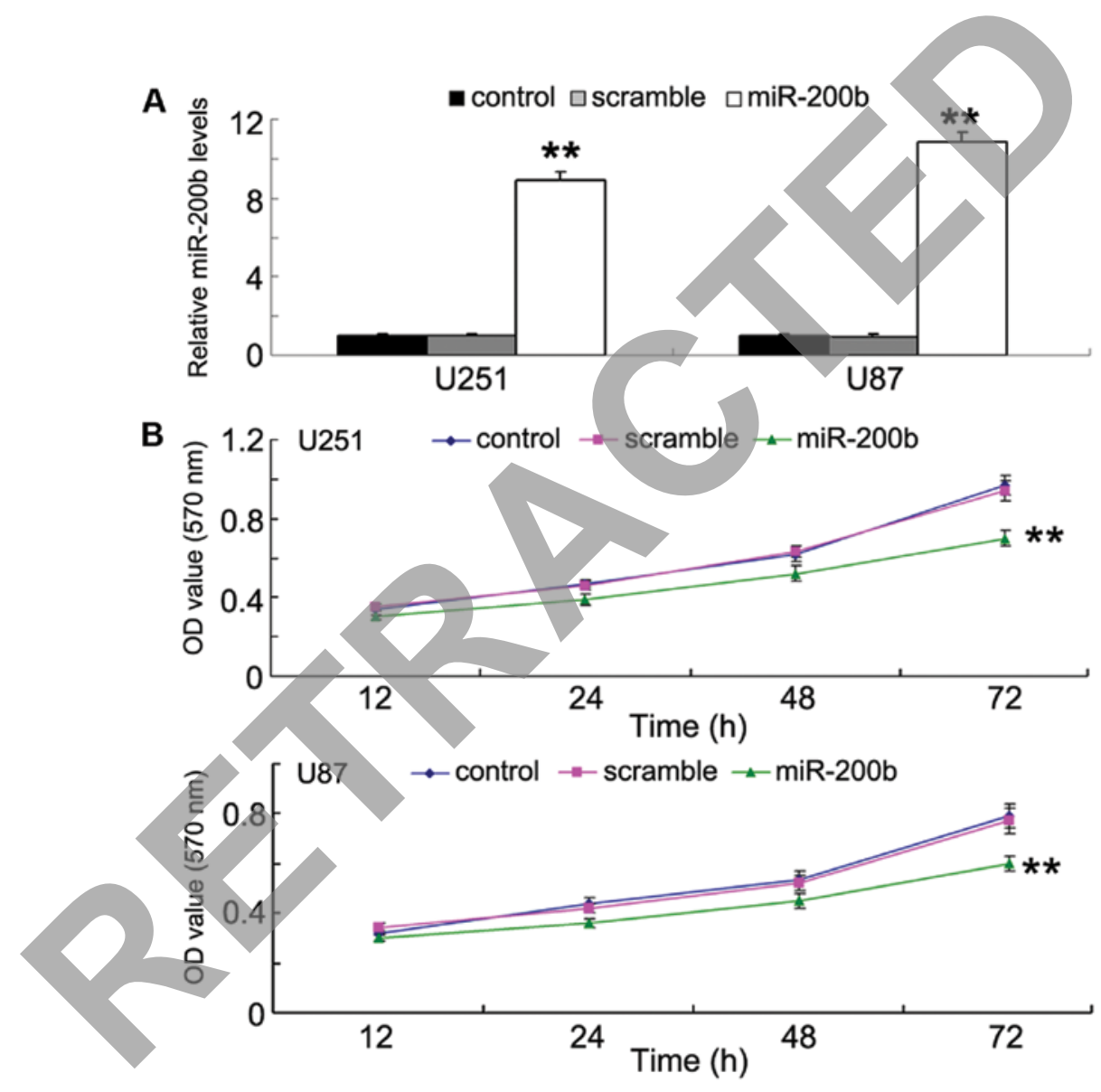

Figure 2. miR-200b inhibits proliferation of U251 and U87 cells. (A) Real-time RT-PCR was conducted to determine the relative miR-200b level in glioma U251 and U87 cells transfected with miR-200b mimic or scramble miR mimic. (B) MTT assay was performed to determine cell proliferation in U251 (upper) and U87 (lower) cells. Non-transfected U251 and U87 cells were used as control. ${ }^{* *} \mathrm{P}<0.01$ vs. control.

Overexpression of miR-200b notably suppresses cell proliferation, migration, invasion and EMT of glioma cells. We further transfected U251 and U87 cells with miR-200b mimic. After transfection, the expression level of miR-200b was significantly upregulated compared to the control group (Fig. 2A). We then performed MTT assay to determine the cell proliferation in each group, and showed that upregulation of miR-200b significantly reduced U251 and U87 cell proliferation (Fig. 2B). Moreover, wound healing assay and Transwell assay were conducted to examine the cell migration and invasion. Overexpression of miR-200b markedly suppressed U251 and U87 cell migration and invasion, compared to the control group, respectively (Fig. 3A and B). We further determine the levels of EMT-related proteins N-cadherin, E-cadherin and vimentin in each group, and showed that upregulation of miR-200b led to increased expression of E-cadherin and decreased $\mathrm{N}$-cadherin and vimentin, when compared to the control group (Fig. 3C). Taken together, we suggest that overexpression of miR-200b can inhibit cell proliferation, migration, invasion and EMT of glioma cells.

ZEB2 is a direct target gene of miR-200b and its expression is negatively regulated by miR-200b in glioma cells. We further used three computational algorithms, PicTar, TargetScan and 
A

U251

U87
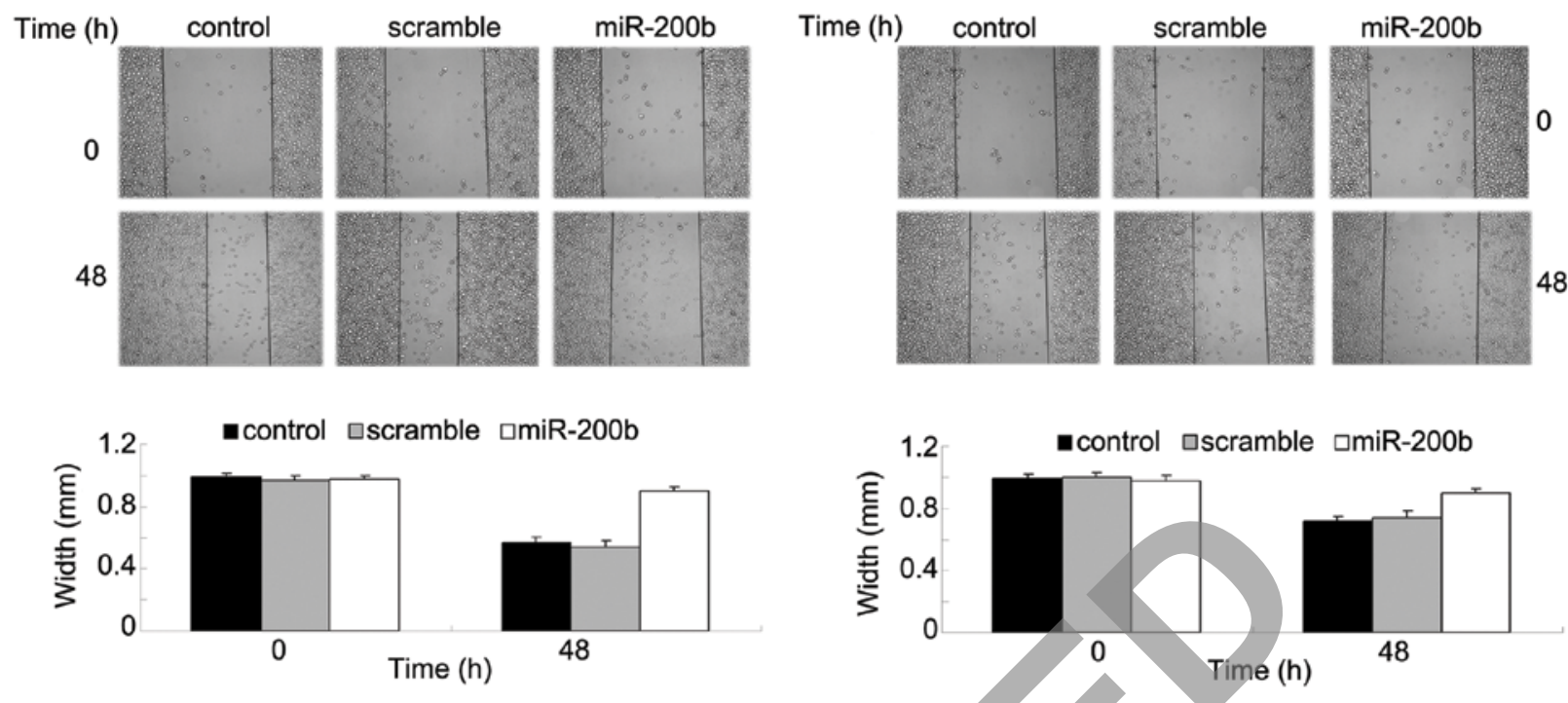

B

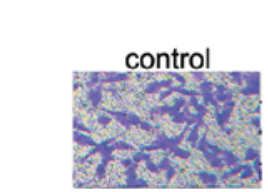

U251
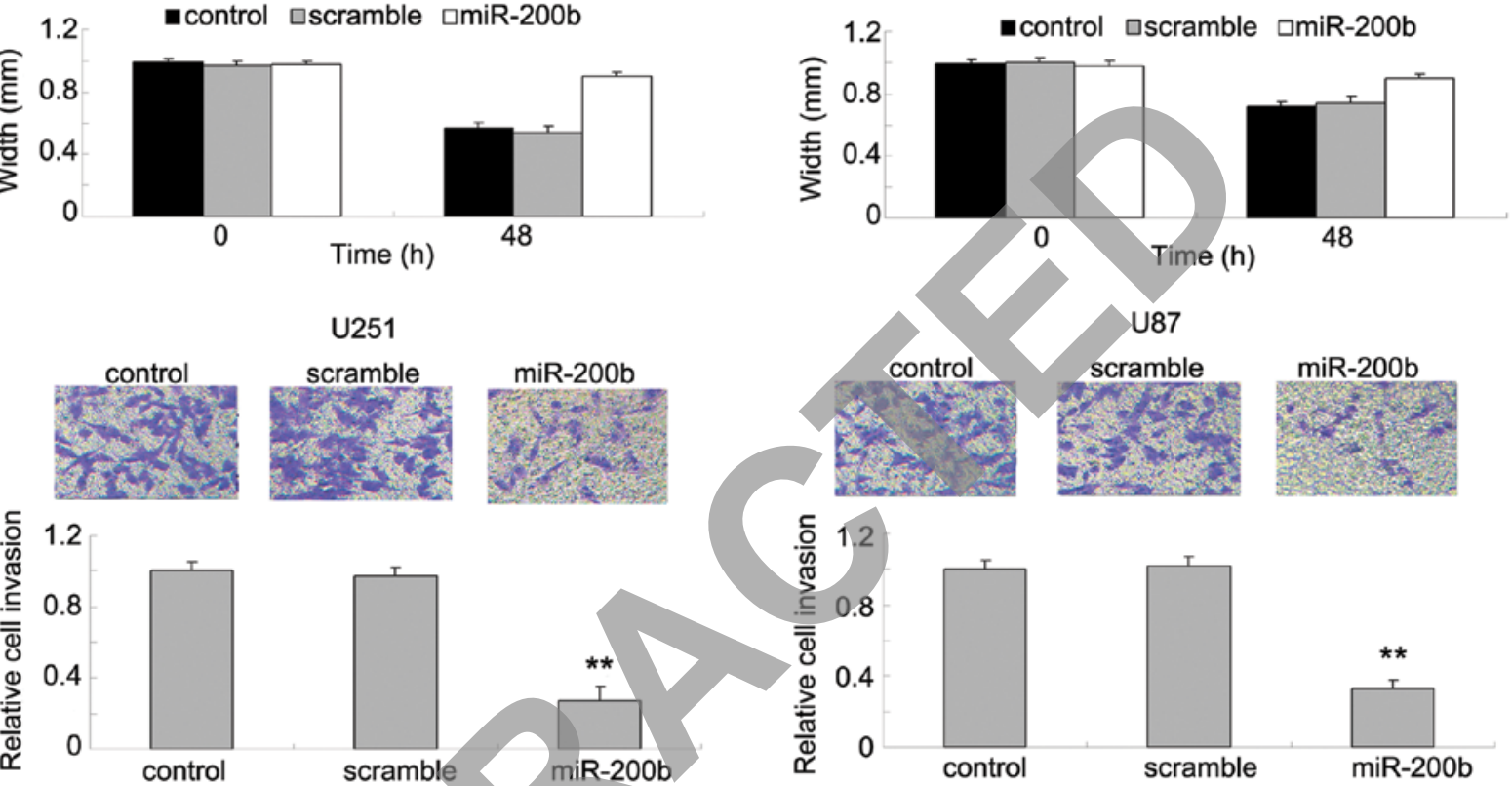

C
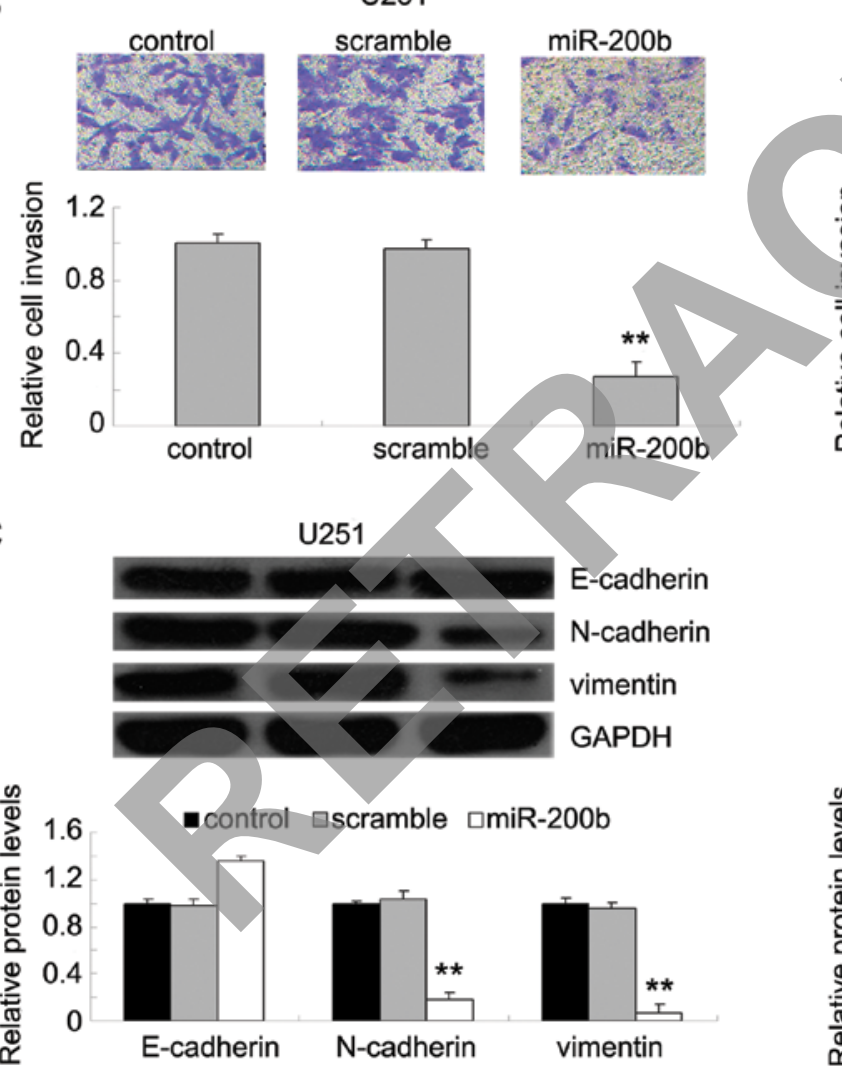

control

U87
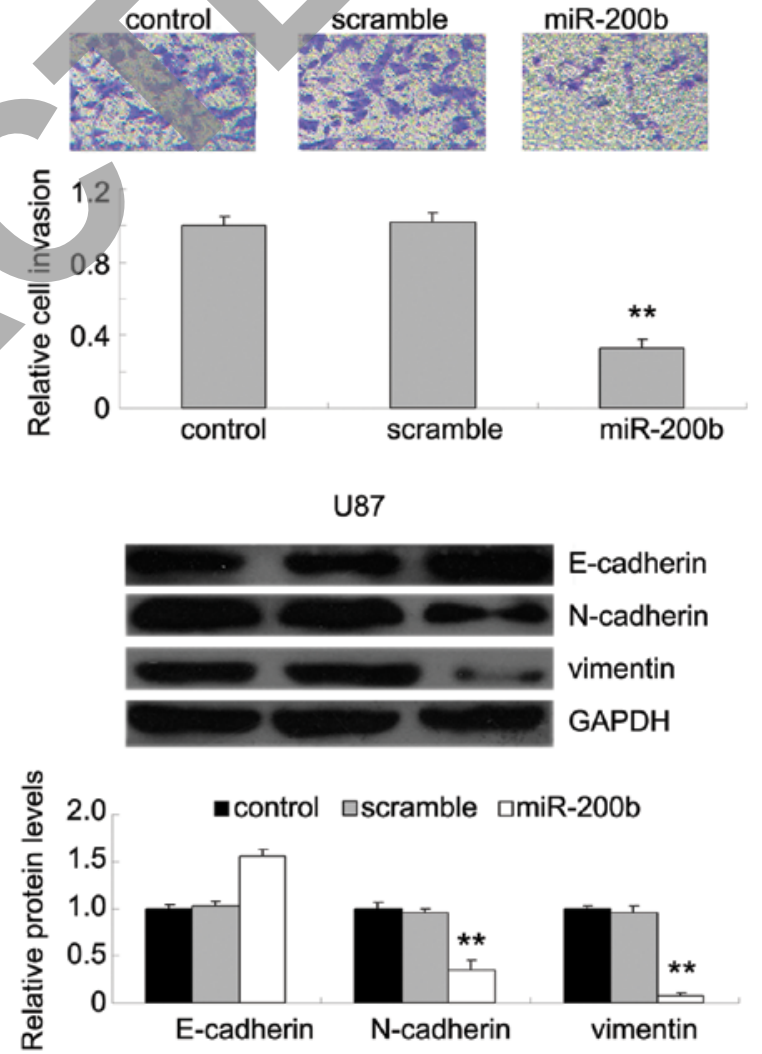

Figure 3. miR-200b inhibits invasive ability of U251 and U87 cells and regulates EMT-related gene expression. (A and B) Wound healing assay and Transwell assay were performed to determine cell migration and invasion in glioma U251 and U87 cell transfected with miR-200b mimic or scramble miR mimic, respectively. (C) Western blot analysis was conducted to examine the protein expression in each group. GAPDH was used as internal reference. Non-transfected U251 and U87 cells were used as control. ${ }^{* *} \mathrm{P}<0.01$ vs. control.

miRanda, to search for potential target genes of miR-200b. ZEB2 was predicted to be a target gene of miR-200b, and perfect base pairing was observed between the seed sequence of mature miR-200b and the 3'UTR of ZEB2 mRNA (Fig. 4A). To verify this predication, we subcloned the wild-type or mutant of ZEB2 3'UTR into a luciferase reporter vector, respectively (Fig. 4B). Co-transfection with pMiR-ZEB2 plasmid and miR-200b mimic resulted in significant decrease in the lucif- erase activity; however, co-transfection with pMiR-Mut ZEB2 plasmid and miR-200b mimic did not change the luciferase activity (Fig. 4C), indicating that miR-200b can directly bind to the 3'UTR of ZEB2 mRNA. We further determined the protein level of ZEB2 in U251 and U87 cells after transfection with miR-200b mimic. Overexpression of miR-200b significantly suppressed the protein expression of ZEB2 in glioma U251 and U87 cells (Fig. 4D). Therefore, we demonstrate that 


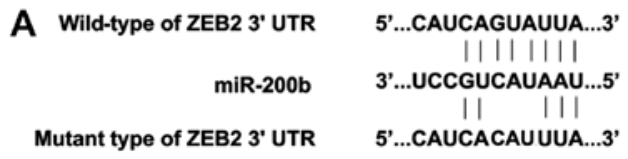

C

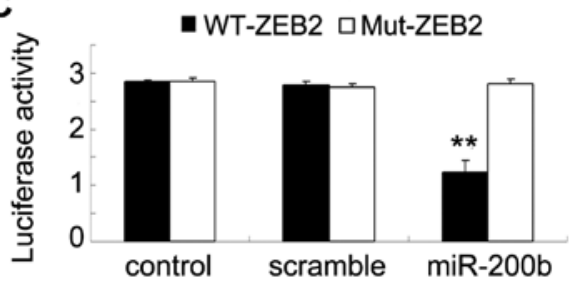

D

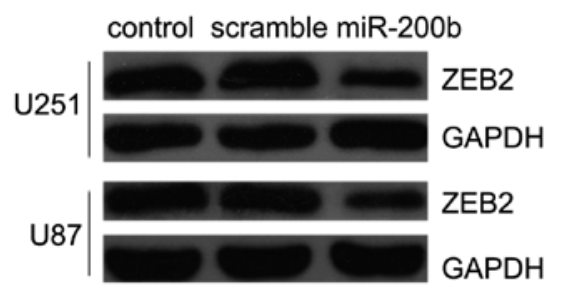

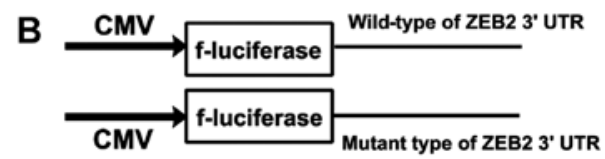
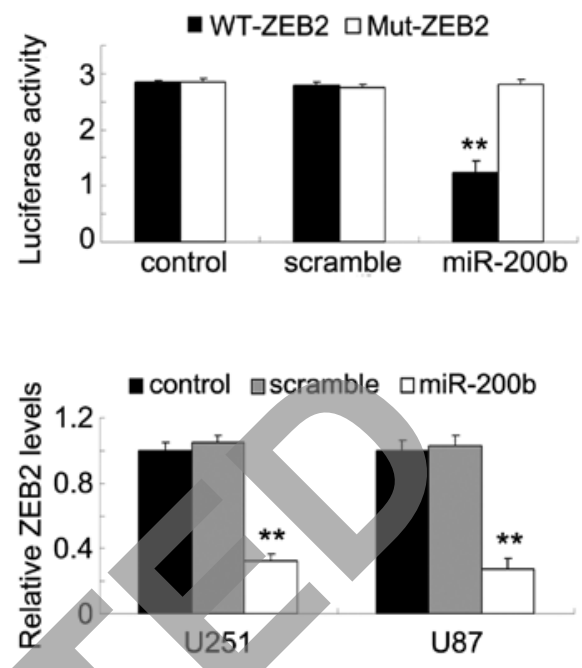

Figure 4. miR-200b directly targets ZEB2. (A) The seed sequences of miR-200b in the wild-type and mutant 3'UTR of ZEB2 are indicated. (B) Representation of the vectors containing the wild-type or mutant type 3'UTR of ZEB2 used in the Luciferase assay. (C) The luciferase activity was notably decreased in glioma U251 and U87 cells co-transfected with miR-200b mimics and pMiR-ZEB2, but unaltered in U251 and U87 cells co-transfected with miR-200b mimics and pMiR-Mut ZEB2. Control: cells only transfected with pMiR-ZEB2 or pMiR-Mut ZEB2, respectively. NC, cells co-transfected with scramble miR and pMiR-ZEB2 or pMiR-Mut ZEB2, respectively. ${ }^{* *} \mathrm{P}<0.01$ vs. control. (D) Western blot analysis was conducted to examine the protein expression of ZEB2 in U251 and U87 cells transfected with miR-200b mimic or scramble miR mimic. GAPDH was used as internal reference. Non-transfected U251 and U87 cells were used as control. ${ }^{* *} \mathrm{P}<0.01$ vs. control.
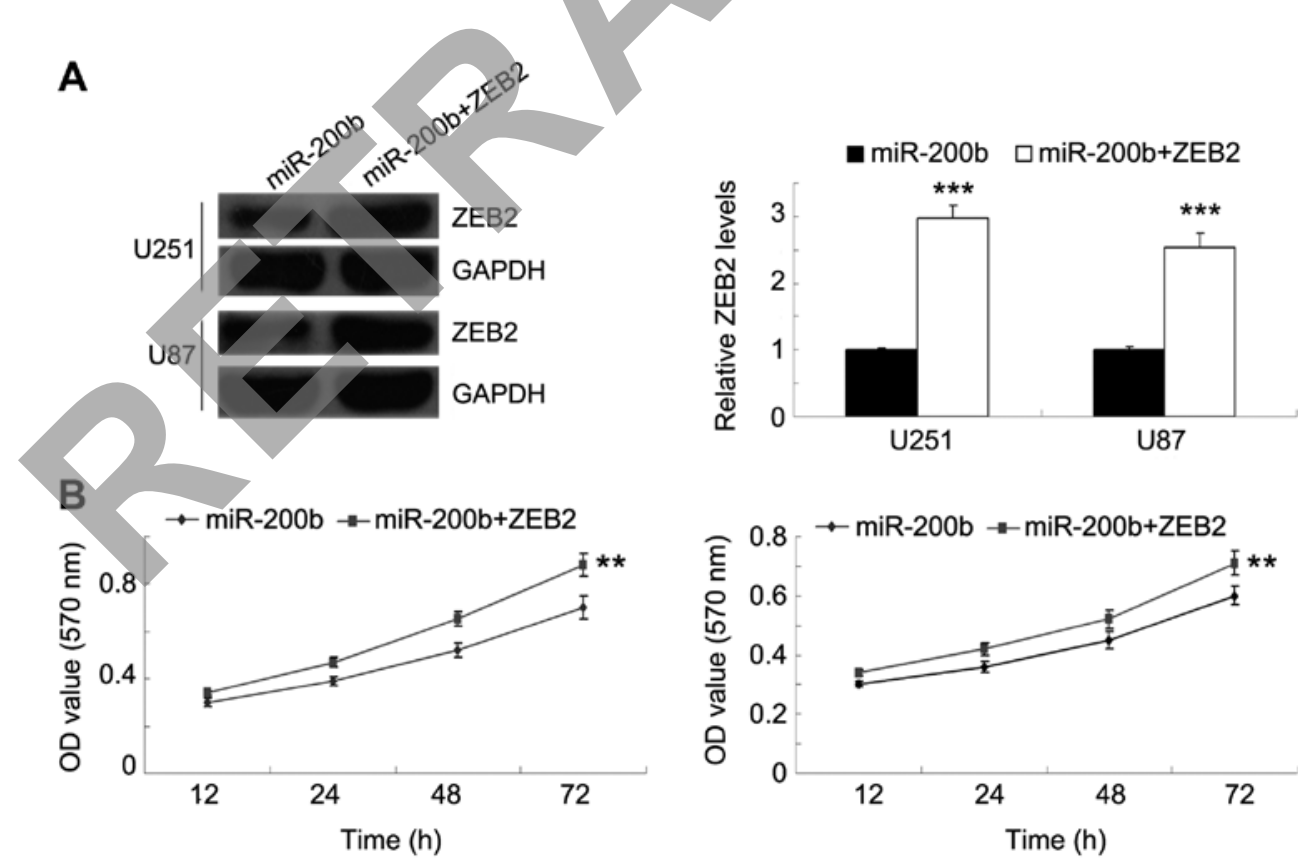

Figure 5. Overexpression of ZEB2 reverses the effects of miR-200b on glioma cell proliferation. (A) Western blot analysis was conducted to examine the protein expression of ZEB2 in U251 and U87 cells transfected with miR-200b mimic, or co-transfected with miR-200b mimic and ZEB2 plasmid, respectively. GAPDH was used as internal reference. (B) MTT assay were performed to determine cell proliferation in each group. ${ }^{* *} \mathrm{P}<0.01 \mathrm{vs}$. miR-200b.

miR-200b negatively regulates the protein expression of ZEB2 via directly binding to the 3'UTR of ZEB2 mRNA in glioma cells.

Overexpression of ZEB2 reverses the suppressive effects of miR-200b overexpression on the malignant phenotypes of glioma cells. We further studied whether ZEB2 was involved in miR-200b-mediated inhibition of cell proliferation, migration, invasion and EMT in glioma cells. miR-200b-overexpressing U251 and U87 cells were transfected with pcDNA3.1-ZEB2 plasmid. After transfection, the decreased protein level of ZEB2 was significantly reversed (Fig. 5A). After that, we 
A
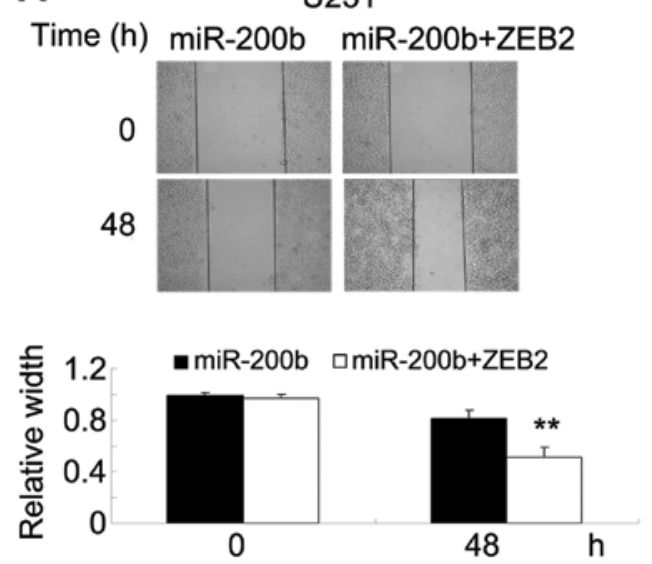

B

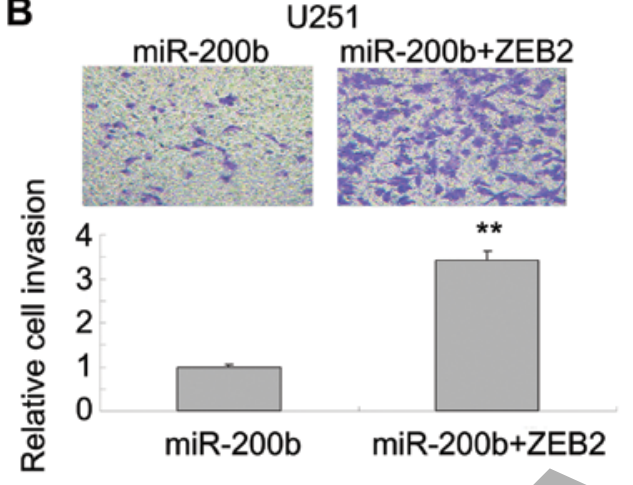

C

U251

miR-200b miR-200b+ZEB2

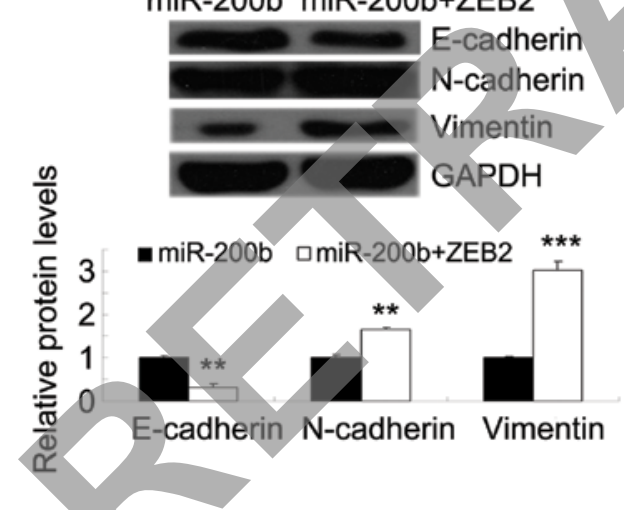

U87

Time (h) miR-200b miR-200b+ZEB2

0
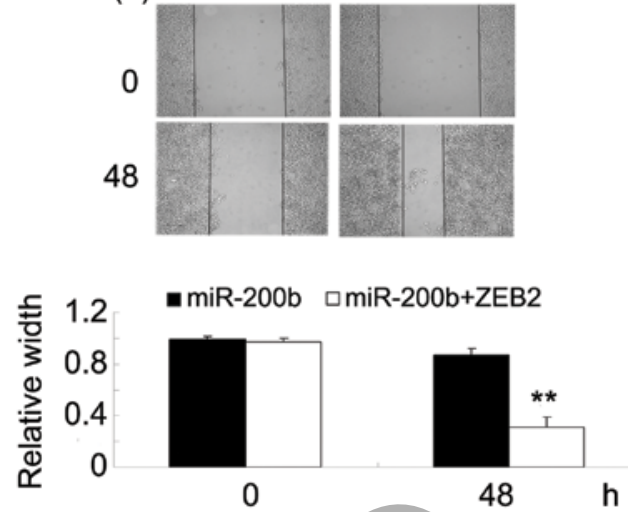

48

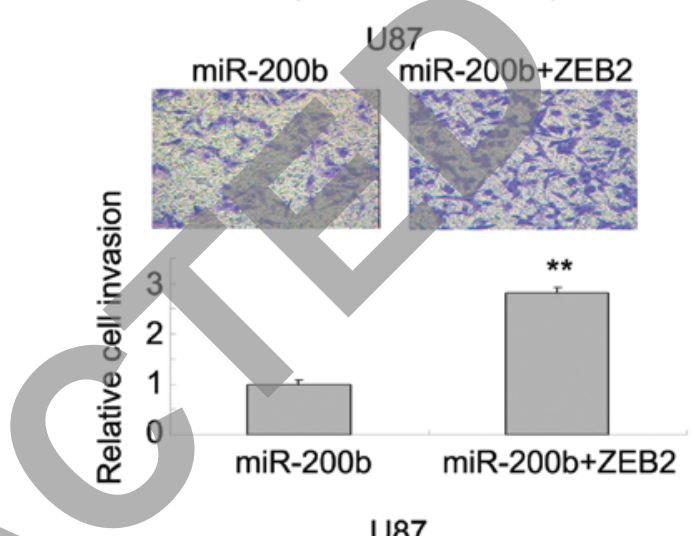

U 87

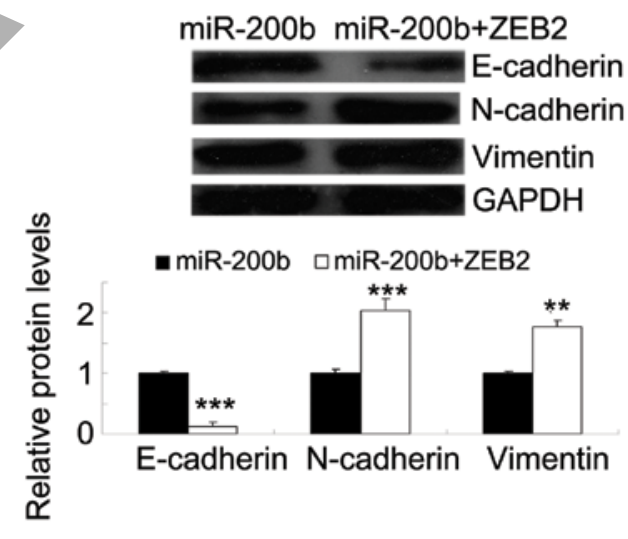

Figure 6. Overexpression of ZEB2 reverses the effects of miR-200b on glioma cell invasion and EMT. (A and B) Wound healing assay and Transwell assay were performed to determine cell migration and invasion in glioma U251 and U87 cell transfected with miR-200b mimic, or co-transfected with miR-200b mimic and ZEB2 plasmid, respectively. (C) Western blot analysis was conducted to examine the protein expression in each group. GAPDH was used as internal reference. ${ }^{* *} \mathrm{P}<0.01$ vs. miR-200b.

compared the malignant phenotypes between glioma cells (U87 and U251) transfected with miR-200b mimic, and cells co-transfected with miR-200b mimic and ZEB2 plasmid. Our data showed that the cell proliferation (Fig. 5B), migration (Fig. 6A) and invasion (Fig. 6B) were all higher in U87 and U251 cells co-transfected with miR-200b mimic and ZEB2 plasmid, when compared to U87 and U251 cells only transfected with miR-200b mimic. Besides, the E-cadherin was downregulated while $\mathrm{N}$-cadherin and vimentin were upregulated in glioma cells co-transfected with miR-200b mimic and ZEB2 plasmid, when compared to U87 and U251 cells only transfected with miR-200b mimic (Fig. 6C), suggesting that the EMT was upregulated after restoration of ZEB2. Taken together, we indicate that restoration of ZEB2 effectively rescues the suppressive effects of miR-200b overexpression on the malignant phenotypes of glioma cells.

miR-200b inhibits the growth of glioma in nude mice. We then examined the effects of miR-200b upregulation on the growth of human glioma xenograft in nude mice. We cloned the miR-200b to pLVX-IRES-ZsGreen1 vector to construct the pYr-LVX-miR-200b lentiviral plasmid. Then, U251 cells were stably transfected with pYr-LVX-miR-200b lentiviral plasmid. In the control group, U251 cells were stably transfected with the blank pLVX-IRES-ZsGreen1 vector. After transfection, the expression level of miR-200b was significantly increased compared to the control group (Fig. 7A). Nude mice were subcutaneously implanted with U251 cells stably transfected 

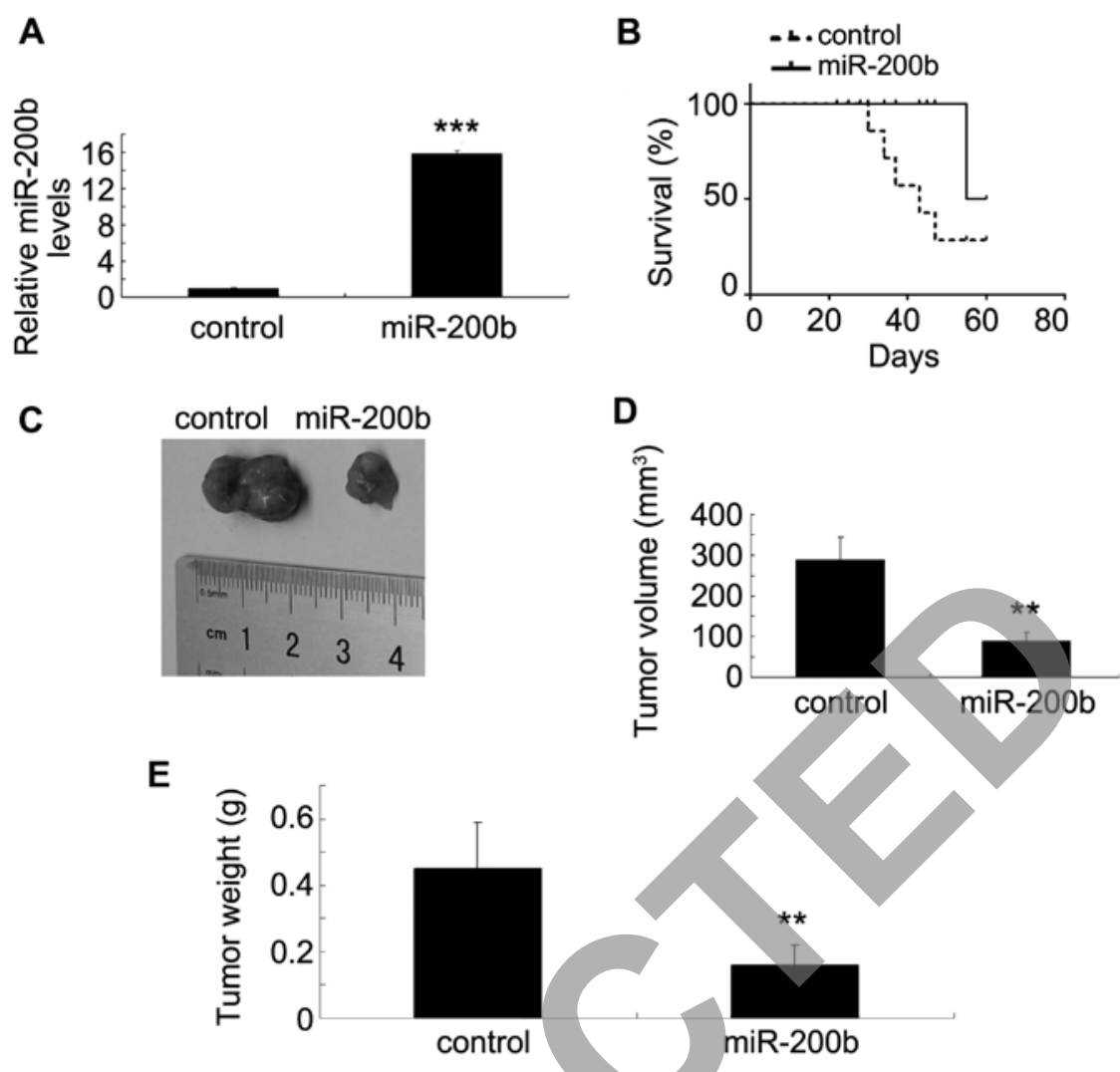

Figure 7. miR-200b suppresses glioma growth in vivo. (A) U251 cells were stably transfected with the blank pLVX-IRES-ZsGreen1 vector as control or pYr-LVX-miR-200b lentiviral plasmid, and real-time RT-PCR was conducted to examine the miR-200b level in each group. (B) Nude mice were subcutaneously implanted with U251 cells stably transfected with pYr-LVX-miR-200b lentiviral plasmid or blank pLVX-IRES-ZsGreen1 vector, respectively. The survival curve is shown. (C) The nude mice in each group were sacrificed 60 days after implantation, and the glioma xenograft was obtained. (D and E) The tumor volume and weight were calculated. ${ }^{* *} \mathrm{P}<0.01$ ys. control.

with pYr-LVX-miR-200b lentiviral plasmid or blank pLVXIRES-ZsGreen1 vector, respectively. After implantation, the tumor gradually grew, and several mice gradually died with the tumor growth. As indicated in Fig. 7B, mice in the control group died within 60 days after implantation, while only 1 mouse in the miR-200b-overexpressing died within 60 days after implantation, indicating that overexpression of miR-200b in U251 cells effectively protected nude mice from tumor-induced death. The surviving mice were sacrificed 60 days after implantation, and the glioma xenograft was obtained (Fig. 7C). As indicated in Fig. 7D and E, the tumor volume and weight in the miR-200b-overexpressing group were markedly higher, when compared with those in the control group. These data suggest that miR-200b can inhibit the growth of glioma in nude mice.

\section{Discussion}

A variety of miRs have been reported to be involved in glioma pathogenesis. For instance, miR-124 can inhibit glioma cell migration and invasion by targeting ROCK1 (21). miR-21 promotes glioma invasion by targeting matrix metalloproteinase (MMP) inhibitors (22). Besides, miR-145 functions as a tumor suppressor in glioma cells by targeting Sox 9 and adducin 3 (23). Recently, several studies revealed the potential role of miR-200b in glioma. Peng et al reported that miR-200b inhibited glioma cell growth via inhibition of CREB1 expression (12). Further two studies reported that decreased miR-200b level was an independent unfavorable prognostic factor for glioma patients $(10,11)$. In addition, DNMT1 and EZH2 were found to mediate the methylation silence of miR-200b and thus promote glioma progression (24). However, the detailed role of miR-200b in glioma malignant progress, as well as the underlying mechanisms, still remain largely unclear. In this study, our data showed that miR-200b was frequently downregulated in glioma tissue specimens, when compared to normal brain tissues. Moreover, we found that decreased miR-200b expression was correlated with malignant progression of glioma, suggesting that miR-200b acts as a tumor suppressor in glioma.

We investigated the function of miR-200b in glioma. Our data showed that the re-introduction of miR-200b significantly inhibited cell proliferation, migration, invasion and EMT of glioma cells in vitro. Accordingly, we suggest that the decreased expression of miR-200b contributes to the advanced progression of human glioma. Similar findings were also reported in other types of human cancers. For instance, Williams et al found that miR-200b inhibited growth, EMT and metastasis of prostate cancer (25). Yu et al found that miR-200b suppressed cell proliferation, migration and enhanced chemosensitivity in prostate cancer by regulating Bmi-1 (26). On the contrary, however, miR-200b was also reported to be an onco-miR that targets the tumor suppressor PTEN in endometrioid endometrial carcinoma (27), suggesting that the role of miR-200b is tumor-specific. 
We further identified ZEB2 as a direct target gene of miR-200b by conducting luciferase reporter assay, and found that miR-200b negatively mediated the protein expression of ZEB2 in glioma cells. ZEB2 is a member of the deltaEF-1 family of two-handed zinc-finger factors, and acts as a transcriptional factor. Recent studies have suggested that deregulation of ZEB2 is associated with the development and progression of multiple types of human cancers, including breast (28), gastric (29), renal (30), pancreatic (31), head and neck (32), glioma (33), hepatocellular (34), ovarian (35), squamous and non-small cell lung carcinomas (36). Moreover, our previous study revealed an oncogenic role of ZEB2 in glioma (18). We found that ZEB2 was significantly upregulated in glioma tissues compared to normal brain tissues, and high protein levels of ZEB2 were positively correlated with malignant progression of glioma. We further found that knockdown of ZEB2 suppressed cell proliferation, migration, invasion and EMT, while induced cell apoptosis and cell cycle arrest in glioma cells (18). Therefore, ZEB2 acts as an oncogene in glioma. In the present study, we found that overexpression of ZEB2 significantly rescued the suppressive effects of miR-200b upregulation on cell proliferation, migration, invasion and EMT in glioma cells. Accordingly, ZEB2 is involved in miR-200b-mediated malignant phenotypes of glioma cells. In addition, several other studies have also demonstrated that ZEB2 is a direct target of miR-200b and acts as a downstream effector in different cell types. For instance, Kurashige et al reported that miR-200b suppressed cell proliferation, invasion, and migration by directly targeting ZEB2 in gastric carcinoma (37). Fang et al (38) found that miR-200b inhibited multi-drug resistance of small cell lung cancer via targeting ZEBB2. Therefore, this study expands the understanding of the relationship between miR-200b and ZEB2 in human cancers.

Finally, our in vivo study showed that overexpression of miR-200b significantly inhibited tumorigenesis as well as the tumor growth of glioma cells, and effectively protected nude mice from tumor-induced death. These data further indicate that miR-200b indeed acts as a tumor suppressor in glioma.

In conclusion, this study investigated the suppressive role of miR-200b in the growth and metastasis of glioma cells, and suggests that miR-200b acts as a novel tumor suppressor miRNA in glioma. miR-200b has suppressive effects on the proliferation, migration, invasion and EMT of glioma cells, partly at least, via targeting ZEB2. The present study provides novel insights into the mechanism underlying the development and progression of glioma, and suggests that miR-200b may become a promising therapeutic candidate for glioma.

\section{Acknowledgements}

This study was supported by the National Natural Science Foundation of China (grant no. 81400938)

\section{References}

1. Goodenberger ML and Jenkins RB: Genetics of adult glioma Cancer Genet 205: 613-621, 2012.

2. Agarwal S, Sane R, Oberoi R, Ohlfest JR and Elmquist WF: Delivery of molecularly targeted therapy to malignant glioma, a disease of the whole brain. Expert Rev Mol Med 13: e17, 2011.
3. Stewart LA: Chemotherapy in adult high-grade glioma: A systematic review and meta-analysis of individual patient data from 12 randomised trials. Lancet 359: 1011-1018, 2002.

4. Zhu VF, Yang J, Lebrun DG and Li M: Understanding the role of cytokines in Glioblastoma Multiforme pathogenesis. Cancer Lett 316: 139-150, 2012.

5. Pulkkanen KJ and Yla-Herttuala S: Gene therapy for malignant glioma: Current clinical status. Mol Ther 12: 585-598, 2005.

6. Moss EG: MicroRNAs: Hidden in the genome. Curr Biol 12: R138-R140, 2002.

7. Choi E, Choi E and Hwang KC: MicroRNAs as novel regulators of stem cell fate. World J Stem Cells 5: 172-187, 2013.

8. Lujambio A, Calin GA, Villanueva A, Ropero S, SanchezCespedes M, Blanco D, Montuenga LM, Rossi S, Nicoloso MS, Faller WJ, et al: A microRNA DNA methylation signature for human cancer metastasis. Proc Natl Acad Sci USA 105: 13556-13561, 2008.

9. Calin GA and Croce CM: MicroRNA signatures in human cancers. Nat Rev Cancer 6: 857-866, 2006.

10. Men D, Liang Y and Chen L: Decreased expression of microRNA-200b is an independent unfavorable prognostic factor for glioma patients. Cancer Epidemiol 38: 152-156, 2014.

11. Liu Q, Tang H, Liu X, Liao Y, Li H, Zhao Z, Yuan X and Jiang W: miR-200b as a prognostic factor targets multiple members of RAB family in glioma. Med Oncol 31: 859, 2014.

12. Peng B, Hu S, Jun Q, Luo D, Zhang X, Zhao H and Li D: MicroRNA-200b targets CREB1 and suppresses cell growth in human malignant glioma. Mol Cell Biochem 379: 51-58, 2013.

13. Remacle JE, Kraft H, Lerchner W, Wuytens G, Collart C, Verschueren K, Smith JC and Huylebroeck D: New mode of DNA binding of multi-zinc finger transcription factors: deltaEF1 family members bind with two hands to two target sites. EMBO J 18: 5073-5084, 1999.

14. Beuran M, Negoi I, Paun S, Ion AD, Bleotu C, Negoi RI and Hostiuc S: The epithelial to mesenchymal transition in pancreatic cancer: A systematic review. Pancreatology 15: 217-225, 2015.

15. Zielinska HA, Bahl A, Holly JM and Perks CM: Epithelialto-mesenchymal transition in breast cancer: A role for insulin-like growth factor I and insulin-like growth factor-binding protein 3 ? Breast Cancer (Dove Med Press) 7: 9-19, 2015.

16. Ye Y, Xiao Y, Wang W, Yearsley K, Gao JX, Shetuni B and Barsky SH: ERalpha signaling through slug regulates E-cadherin and EMT. Oncogene 29: 1451-1462, 2010.

17. Nam EH, Lee Y, Park YK, Lee JW and Kim S: ZEB2 upregulates integrin alpha5 expression through cooperation with Sp1 to induce invasion during epithelial-mesenchymal transition of human cancer cells. Carcinogenesis 33: 563-571, 2012.

18. Qi S, Song Y, Peng Y, Wang H, Long H, Yu X, Li Z, Fang L, Wu A, Luo W, et al: ZEB2 mediates multiple pathways regulating cell proliferation, migration, invasion, and apoptosis in glioma. PLoS One 7: e38842, 2012.

19. Liu Z, Long X, Chao C, Yan C, Wu Q, Hua S, Zhang Y, Wu A and Fang W: Knocking down CDK4 mediates the elevation of let-7c suppressing cell growth in nasopharyngeal carcinoma. BMC Cancer 14: 274, 2014.

20. Liu B, Che W, Xue J, Zheng C, Tang K, Zhang J, Wen J and $\mathrm{Xu}$ Y: SIRT4 prevents hypoxia-induced apoptosis in $\mathrm{H} 9 \mathrm{c} 2$ cardiomyoblast cells. Cell Physiol Biochem 32: 655-662, 2013.

21. An L, Liu Y, Wu A and Guan Y: microRNA-124 inhibits migration and invasion by down-regulating ROCK1 in glioma. PLoS One 8: e69478, 2013.

22. Gabriely G, Wurdinger T, Kesari S, Esau CC, Burchard J, Linsley PS and Krichevsky AM: MicroRNA 21 promotes glioma invasion by targeting matrix metalloproteinase regulators. Mol Cell Biol 28: 5369-5380, 2008 .

23. Rani SB, Rathod SS, Karthik S, Kaur N, Muzumdar D and Shiras AS: MiR-145 functions as a tumor-suppressive RNA by targeting Sox 9 and adducin 3 in human glioma cells. Neuro Oncol 15: 1302-1316, 2013.

24. Ning X, Shi Z, Liu X, Zhang A, Han L, Jiang K, Kang C and Zhang Q: DNMT1 and EZH2 mediated methylation silences the microRNA-200b/a/429 gene and promotes tumor progression. Cancer Lett 359: 198-205, 2015.

25. Williams LV, Veliceasa D, Vinokour E and Volpert OV: miR-200b inhibits prostate cancer EMT, growth and metastasis. PLoS One 8: e83991, 2013.

26. Yu J, Lu Y, Cui D, Li E, Zhu Y, Zhao Y, Zhao F and Xia S: miR-200b suppresses cell proliferation, migration and enhances chemosensitivity in prostate cancer by regulating Bmi-1. Oncol Rep 31: 910-918, 2014 
27. Yoneyama K, Ishibashi O, Kawase R, Kurose K and Takeshita T: miR-200a, miR-200b and miR-429 are onco-miRs that target the PTEN gene in endometrioid endometrial carcinoma. Anticancer Res 35: 1401-1410, 2015.

28. Zhang Z, Yang C, Gao W, Chen T, Qian T, Hu J and Tan Y: FOXA2 attenuates the epithelial to mesenchymal transition by regulating the transcription of E-cadherin and ZEB2 in human breast cancer. Cancer Lett 361: 240-250, 2015.

29. Li H, Xu L, Zhao L, Ma Y, Zhu Z, Liu Y and Qu X: Insulin-like growth factor-I induces epithelial to mesenchymal transition via GSK-3beta and ZEB2 in the BGC-823 gastric cancer cell line. Oncol Lett 9: 143-148, 2015.

30. Chen Z, Tang ZY, He Y, Liu LF, Li DJ and Chen X: miRNA-205 is a candidate tumor suppressor that targets ZEB2 in renal cell carcinoma. Oncol Res Treat 37: 658-664, 2014

31. Usova EV, Kopantseva MR, Kostina MB, Van'Kovich AN, Egorov VI and Kopantsev EP: Expression of the ZEB2 gene in pancreatic stromal cells in pancreatic ductal adenocarcinoma, pancreatitis, and normal state. Dokl Biol Sci 448: 61-64, 2013.

32. Chu PY, Hu FW, Yu CC, Tsai LL, Yu CH, Wu BC, Chen YW, Huang PI and Lo WL: Epithelial-mesenchymal transition transcription factor ZEB1/ZEB2 co-expression predicts poor prognosis and maintains tumor-initiating properties in head and neck cancer. Oral Oncol 49: 34-41, 2013.
33. Xia M, Hu M, Wang J, Xu Y, Chen X, Ma Y and Su L: Identification of the role of Smad interacting protein 1 (SIP1) in glioma. J Neurooncol 97: 225-232, 2010.

34. Yang X, Wang J, Qu S, Zhang H, Ruan B, Gao Y, Ma B, Wang X, Wu N, Li X, et al: MicroRNA-200a suppresses metastatic potential of side population cells in human hepatocellular carcinoma by decreasing ZEB2. Oncotarget 6: 7918-7929, 2015.

35. Guo F, Cogdell D, Hu L, Yang D, Sood AK, Xue F and Zhang W: miR-101 suppresses the epithelial-to-mesenchymal transition by targeting ZEB1 and ZEB2 in ovarian carcinoma. Oncol Rep 31 2021-2028, 2014.

36. You J, Li Y, Fang N, Liu B, Zu L, Chang R, Li X and Zhou Q: MiR-132 suppresses the migration and invasion of lung cancer cells via targeting the EMT regulator ZEB2. PLoS One 9: e91827, 2014.

37. Kurashige J, Kamohara H, Watanabe M, Hiyoshi Y, Iwatsuki M, Tanaka Y, Kinoshita K, Saito S, Baba Y and Baba H: MicroRNA-200b regulates cell proliferation, invasion, and migration by directly targeting ZEB2 in gastric carcinoma. Ann Surg Oncol 19 (Suppl 3): S656-S664, 2012.

38. Fang S, Zeng X, Zhu W, Tang R, Chao Y and Guo L: Zinc finger E-box-binding homeobox 2 (ZEB2) regulated by miR-200b contributes to multi-drug resistance of small cell lung cancer. Exp Mol Pathol 96: 438-444, 2014. 